\title{
Involvement of Cloud Computing and IoT in the Field of Health Care
}

\author{
Hareesh Kumar, \\ Department of Computer Science and Engineering, \\ Sachdeva Institute of Technology, Mathura (SIT Mathura), \\ Dr. A P J Abdul Kalam Technical University, \\ Lucknow, Uttar Pradesh, \\ India.
}

Abstract- The rapid growth of the Internet of Things (IoT) and cloud computing is finding is a big part of our lifestyle, planning to upgrade personal satisfaction by partnering with various shrewd gadgets, innovations, and applications. Generally, the fields of IoT and cloud computing considers the mechanization of everything around the world. Presently scientists have discovered that there is a reasonably big utilization of IoT and cloud in the field of the health care industry. In between the panoply of uses empowered by the Internet of Things (IoT), brilliant and associated health care services play a significant role. The arrangement of network sensors, either exhausted on the body or incorporated into our living environment which makes the processing of information demonstrative of our physical and emotional well-being. Such data can achieve a positive disruptive shift in the human services, collected consistently, totaled, and adequately mined. This study exhibits the significance of the Internet of Things and Cloud in the field of the Healthcare Industry.

Keywords- Internet of Things; Cloud Computing; Cloud of Things; Healthcare and Cloud.

\section{INTRODUCTION}

Cloud computing and the Internet of Things (IoT) are the fundamental innovation to shape smart cities as the elements to provide data and administrations to clients by teaming up and articulating with one another [1]. 
Journal of trends in Computer Science and Smart technology (TCSST) (2021)

Vol.03/ No. 01

Pages: $1-13$

https://www.irojournals.com/tcsst/

DOI: https://doi.org/10.36548/jtcsst.2021.1.001

The beneficiary of the Internet of Things can be huge in the field of medicinal services applications. During the utilization of IoT based restorative administrations, sensors work for screening and quantifying different wellbeing parameters in the human body. These gadgets can be mainly centered over the monitoring condition of a patient when they are distant from everyone else or when they are not hospitalized. Thusly, a consistent input to the authority, relatives, or the patient is provided by them. Various wearable used for detecting gadgets in the market and they are fitted out with medicinal sensors to monitor various requirements, for example, body temperature, pulse, circulatory strain, breath rate, heartbeat and so on.

Healthcare applications create a free-living environment that is conceivable and increasingly appropriate for the elderly and patients with serious health conditions. The use of IoT sensors is efficiently used in the current scenario to monitor and screen well-being conditions and transmit cautions if any phenomenal signs are detected.

In addition, if there is a case of a mild problem, the IoT application has a course of action to administer a drug to patients. IoT-cloud excellently observes the crisis response and idleness for data trading.

The data is transferred among edge servers, cloud, and client gadgets which straightforwardly influences the demonstration. To accomplish this objective edge figuring appeared and distributed computing stays on the edge of the framework to help constrained transmission capacity, dormancy, and system clog. The edge registration was also developed to assist dispersed and virtual innovation to provide cloud and IoT gadgets such as sensors with device support [2].

The majority of the proposed cloud-based IoT medicinal services observing structures have three parts such as information obtaining for utilizing wearable sensors, transmission information which administers constant sending of the information to the server of the social support association in a safe manner, and the cloud preparing for information such as stockpiling, investigation, and perception [3]. For the most part, the e-Healthcare control system consists of: 
Journal of trends in Computer Science and Smart technology (TCSST) (2021)

Vol.03/ No. 01

Pages: $1-13$

https://www.irojournals.com/tcsst/

DOI: $\underline{\text { https://doi.org/10.36548/jtcsst.2021.1.001 }}$

i. A group of sensors for the identification of physiological constraints of patients, whether keen or commonly sensed;

ii. Wireless Body Area Network (WBAN) that is dependable upon IoT correspondence to enable Machine-to-Machine (M2M), and also correspondence in between Elements or empowering doctors to slightly communicate with medical server;

iii. Medical server over the Cloud used for information stockpiling, preparing, and analytical examination;

iv. Medical clinics pertain to doctors (e.g., specialists) who can get data remotely from the medicinal server in the Cloud [4].

These days, the research examination in this field approaches the reconciliation of Cloud Computing and the Internet of Things [5], [6]. This amalgamation is alluded to as Cloud of Things (CoT) which is another worldview used to endeavors the reconciliation of two remarkable and prominent advances such as the Internet of Things and the Cloud, and the advancement of Futuristic opportunity for Internet applications [7].

Although both IoT and Cloud are two unique and autonomous technological developments, there is a need to organize them to complement each other and have the option of assisting with inevitable and omnipresent figures [8][9].

In this article, the main discussion is about the integration of cloud computing and the Internet of Things and further about the importance of cloud computing and finally, about the internet of things in the field of healthcare services. 
Journal of trends in Computer Science and Smart technology (TCSST) (2021)

Vol.03/ No. 01

Pages: $1-13$

https://www.irojournals.com/tcsst/

DOI: https://doi.org/10.36548/jtcsst.2021.1.001

\section{INTEGRATION OF CLOUD COMPUTING AND IOT}

Some specialists have developed a subject known as the Cloud of Things (CoT) to provide reconciliation between the cloud and the IoT [10]. CoT paradigm planned for using the IoT along with the Cloud.

When implementing CoT, Cloud is a middleware that creates a straightforward link between obje cts and customers/applications (i.e. decreases the unpredictability that facilitates the enhancemen t of uses that handle excellent objects) [11]. Cloud will help IoT with its boundless capacity and figuring assets, while IoT allows the Cloud the opportunity to expand its administrations to certifiable objects [12]. Numerous endeavors have been made to advance the pattern toward this combination. Sensor-Cloud is one of the most relevant of these initiatives and is related to the mixing of sensors into the cloud server farm and providing arranged access to sensor information and assets for management [13]. For example, by misusing the joining between Cloud and IoT as pursuits, numerous benefits can be remarkable.

i. Well-organized stockpiling for IoT information by exploiting the Cloud stockpiling nature, i.e., On-request, practically boundless and minimal effort [14], [7].

ii. The incorporation with Cloud improves IoT handling and calculation by including more capacities that are not permitted at the IoT end, and vitality sparing by empowering errand of stacking [15], [7], [12], [16]. Finally, the Cloud model fulfills the needs of IoT through its for all intents and purposes boundless preparing and on-request utilization, which empowers simple investigation of IoT information.

iii. Cloud provides a knowledgeable and easy solution to enable IoT to observe and supervise protests anywhere without a need to impart expensive committed equipment. Besides, it gives an effective arrangement structure a maturing the created information of Things [7].

iv. In the field of IoT, there are some restrictions in numerous regions, for example, versatility, interoperability, and effectiveness because of the high heterogeneity of its gadgets, 
Journal of trends in Computer Science and Smart technology (TCSST) (2021)

Vol.03/ No. 01

Pages: $1-13$

https://www.irojournals.com/tcsst/

DOI: https://doi.org/10.36548/jtcsst.2021.1.001

advancements, and conventions. Cloud can encourage the progression of IoT information gathering and prepare just as facilitate the procedure of incorporation of new things while lessening the expense of the organization and complex information handling [7].

v. CoT provides progress in new magnificent administrations and applications that affect the growth of the Cloud by objects that operates new issues[7][12] when addressing scalability.

\section{SYSTEM ARCHITECTURE}

Knowledge on the real world and possibility of data generated by wearable therapeutic gadgets with associated electronic well-being records can be highly beneficial for parental figures and a strong hotspot for scientists, as they have the ability to collect discreet information and data effectively outside the office dividers [17]. This can be achieved adequately by identifying restorative gadgets that can persistently dissect and securely store well-being-related data to a cloud, which gives unending data advances to extended timeframes, to achieve genuine perceptive social insurance, and even to find conceivable unfortunate propensities. In the relevant well-being frameworks, it is remarkable to understand how a Personal Health Devices (PHD) transmits wellbeing information, how the information is stored by the use of Personal Health Health Record (PHR) and how the information is securely stored on the web by Personal Health Managers (PHM).

A. Personal Health Devices- Here, wearable or not, Personal Health Devices are resources with obligated assets that allow you to securely accumulate, store, preserve, and partition your own and your family's well-being information to a door with the ultimate purpose of capturing, displaying, and transmitting[18]. Low-control correspondence innovation like Bluetooth ought to be a characteristic decision for the organization of individual e-Health frameworks and gadgets. A passage will transfer information for telephone assistance to a human services administration population for the ultimate purpose of additional analysis and usage data from different fields, such as well-being and health, disease control or a free age estimate gadget as shown in Figure 1. The correspondence between a PHD and the door is called a virtual point-to-point relationship [19]. For the most part, PHD articulates the necessary with a solitary passage at a specific moment. 
Journal of trends in Computer Science and Smart technology (TCSST) (2021)

Vol.03/ No. 01

Pages: $1-13$

https://www.irojournals.com/tcsst/

DOI: https://doi.org/10.36548/jtcsst.2021.1.001

Doors will interface with most of the PHDs using different point-to-point associations at the same time[20].

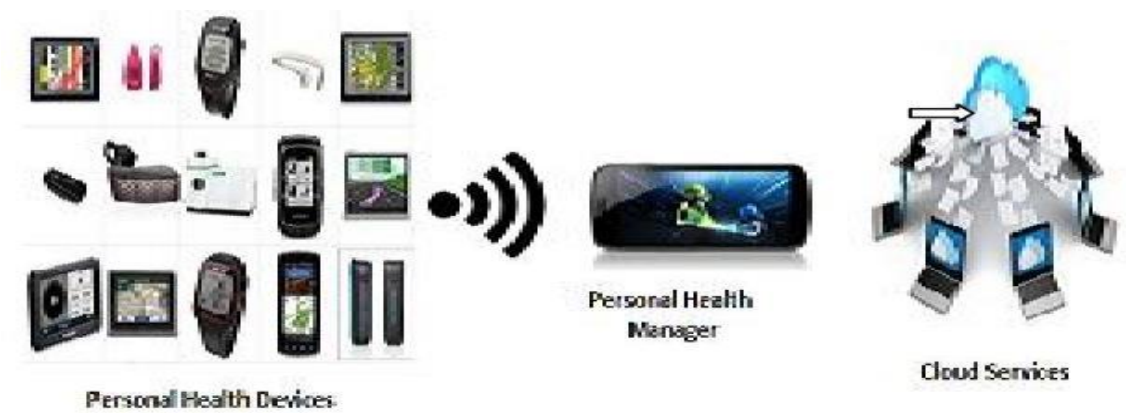

Figure.1. PHD Wireless Transmission [19]

B. Personal Health Record- The PHR is a gadget that is utilized to assemble, track and offer past and current data about the wellbeing or the strength of the individual in your consideration. This data will periodically set aside the money and hassle of rehashing repetitive restorative tests. Despite there is no need to understand the usual approaches, PHR will give providers of restorative consideration more strength in their background of well-being. The particular substance of wellbeing record relies upon the kind of medicinal services have been acquired. Recorded down are reports ordinary to most wellbeing records and extra archives that partner with medical clinic stays or medical procedures [35]. Past activities and besides is more forward-thinking consideration of the development of the structure.

\section{LITERATURE REVIEW}

Cloud Computing (CC) and the Internet of Things (IoT) have mutually provided essentially altered the data innovation industry. At this point, Cloud computing has assisted in the development of proficient applications concerning adaptability, virtualization, unwavering quality and cost costs. Then again, IoT with its inventive components, for example, Radio Frequency Identification (RFID) and sensors could be effective helps in understanding the world objects to accomplish observing and the board in a versatile district. As of now, Cloud and IoT are broadly connected in countless data innovation applications. Notwithstanding, the continuous research in human services toward the combination of Cloud and IoT do not meet the prerequisites. Acquiring this 
Journal of trends in Computer Science and Smart technology (TCSST) (2021)

Vol.03/ No. 01

Pages: $1-13$

https://www.irojournals.com/tcsst/

DOI: https://doi.org/10.36548/jtcsst.2021.1.001

incorporation in the setting of human services can fundamentally add to building effective social insurance applications for overseeing and proficiently observing clinics and patients for asset sharing and cost consumptions.

According to the research work of S. Luo and B. Ren, the author described in the aspects of Cloud and IoT, fragile health care checking observation devices and the data administrations can effectively provide early recognition and treatment of constant illnesses that significantly affect an individual's well being. Such that, IoT body sensors collect the necessary data from a person (i.e. implantable or wearable) and then the data can be decomposed and prepared in the Cloud [21].

The utilization of CoT in the health care space offers new chances to restorative IT foundation and can improve medicinal services administrations mentioned by author L. Catarinucci et al. [22]. In addition, CoT strengthens the types of medicinal services and the essence of real social security benefits by rearranging the way to the imperative knowledge of patients and transmitting them to a therapeutic emphasis for capability and planning purposes on Cloud[7].According to the work of author G. Fortino et al. this can be put forward that the utilization of CoT in Body Sensor Network (BSN) dependent medicinal services help during the time spent away from accumulated information, this is similar to preparing and dissecting them in an adaptable style [16]. With CoT, human services sensors can be overseen productively in a straightforward way just as make any managing hazardous medicinal services benefits that are highly effective mentioned by author M. Aazam and E.-N. Huh [23].

To accomplish productive medicinal services administrations concerning delay-touchy and vitality utilization, haze registering can assume a crucial job by Cloud weight reduction as a localized stockpiling of IoT gadgets and its ability to manage information [24][25][26][26]. Since patients' information is hazardous, guidelines do not enable them to be prepared outside the association of the medicinal service [27]. Fog Computing is expected to fill this hole along these lines by carrying planning capabilities nearer to social insurance suppliers (e.g., clinic). After doing this, a lot of advantages can be obtained, such as decreased idleness and decreased use of vitality, as well as increased use of transfer speed and protection of knowledge. 
Journal of trends in Computer Science and Smart technology (TCSST) (2021)

Vol.03/ No. 01

Pages: $1-13$

https://www.irojournals.com/tcsst/

DOI: https://doi.org/10.36548/jtcsst.2021.1.001

After the implementation of Fog-empowered CoT, the gathered tangible information can be handled and broke down in the neighbourhood passage (keen portal) though doctors can be overcome through the Cloud remotely. Following this methodology reduces information transmission and execution times just as spares vitality. The sorts of medicinal services gadget utilized rely upon the application sending situation. For example, in the portable patient checking organization situation, Smart telephones can be utilized as a WPAN passage for empowering direct association with the WAN through cell systems. During this situation, the sensor gadgets wearable or implantable, converse with the WPAN through the portal (i.e., the Smartphone).

The presence of medicinal services gadgets with the Cloud additionally relies upon the health care application arrangement situation. For instance, a few situations may associate legitimately to the WLAN through Wi-Fi correspondence while in others it might interface utilizing WPAN employing Bluetooth is mentioned author by F. A. Kraemer et al. [27]. Other than the identification and discovery of changes in the condition of patients, advanced cell phone-based portals can effectively provide easy communication between IoT gadgets and the Internet as well as administration of IoT gadgets.

G. Aloi et al. [28], the author planned of an abnormal state structure of a smart phone-driven to help flexible and straightforward interoperability. Due to the expanded pace of constant illnesses in both developed and developing nations are concerned about creating medicinal services activities dependent on ICT advances for providing social insurance administration has developed. According to the work of author F. Ramalho et al. related to the Virtual Cloud Carer (VCC) [26] is a CoT-based venture subsidized by Spanish National R\&D planned to provide creative social insurance administrations to reliant and individuals with serious illnesses issues.

With the implementation of CoT, the VCC task means to increase social and innovative goals that improve the nature of the new administrations being offered to the traditional models. These destinations extend from making stage engineering - that is in charge of social event physiological parameters from anyplace and convey them to the Cloud for capacity and preparing purposes. To 
Journal of trends in Computer Science and Smart technology (TCSST) (2021)

Vol.03/ No. 01

Pages: $1-13$

https://www.irojournals.com/tcsst/

DOI: https://doi.org/10.36548/jtcsst.2021.1.001

support the elderly to do their physical planning procedures and to assist parental figures in a professional way to monitor and screen the elderly remotely.

According to the work of author Danilo, et al. [19] regarding the expanding accessibility of associated Personal Health Devices (PHDs) empowers another sort of data to be accessible on the Internet: wellbeing data. The majority of these gadgets have explicit approaches to associate and share data to the Internet through doors or wellbeing directors, and making vertical arrangements where one gadget just converses with one wellbeing administration. In this unique situation, this article proposes a design that suggests about the utilization of various sorts of wellbeing supervisors however keeping interoperability by the utilization of generally embraced models. The primary commitment of this work is the diffusion of well-being managers in various fields, such as mobile phones and cloud applications, allowing the use of solitary well-being management for different types of PHDs.

\section{CLOUD AND IOT GADGETS FOR HEALTH CARE}

The IoT's empowerment influences are genius gadgets that track and remotely monitor constantly following imaginable and cloud-based administrations that power networks. Numerous applications discuss verbally with the patients in regards to their wellbeing routine [29]. The application conveys warnings to the patients. There is a particular gathering of patients who often practice these devices, one such gathering is those with standard age-related diseases such as blood weights and blood sugars, and patients with heftiness are another gathering[30]. A few instances of each combined with the IoT to help proactive social insurance.

A. Insulin Injection Trackers-Extreme insulin infusion tracker causes diabetic patients to deal with their wellbeing. The infusion tracker is a mechanized top that suits most insulin pens available. It remotely transmits a diabetic's insulin infusion information to an advanced operating application.

B. Prescription Pills-Physician recommended medicine have likewise accompanied the IoT. An ingestible sensor that is a piece of the pill you swallow each day is created which tracks whether a 
Journal of trends in Computer Science and Smart technology (TCSST) (2021)

Vol.03/ No. 01

Pages: $1-13$

https://www.irojournals.com/tcsst/

DOI: https://doi.org/10.36548/jtcsst.2021.1.001

patient is taking his drug on the calendar. At the point, when the pill arrives at the patient's stomach and is divulged to stomach liquid, it transmits a sign through the claim body tissue of the client to a fix on the patient's skin. The fix at that point communicates its information to a PDA, where it can be imparted to doctors and parental figures.

C. Asthma Nitric Oxide (NO) monitor-It is a minor, hand-held gadget that takes ceaseless estimations of NO in a patient's breath, which can help the administration of asthma and other fiery aviation route conditions. At the point, when a patient breathes into the mouthpiece of the gadget, the degrees of NO in their breath are estimated to choose if there are aviation route irritation and the best medicine to treat it. The information that is gathered can be synchronized with electronic therapeutic record frameworks.

D. Hypothermia-Hypothermia locator is a savvy liquid administration gadget that naturally measures pee yield and Core Body Temperature (CBT) for patients whose bladder is embedded with a urinary catheter. By checking the crucial signs, care can be started right on time for heart disappointment, kidney damage, irresistible sickness, sepsis, prostate tumors, diabetes, and consume patients. Estimating CBT can likewise connote contamination or hypothermia. At the point when utilized in medical clinics, this gadget gives fill level and CBT information legitimately to a nursing station or to a screen remotely.

\section{CONCLUSION AND FUTURE WORK}

Progression in empowering brilliant advancements, for example, IoT, enormous information, mist, and distributed computing and so forth., savvy city and applications are expanding zone of research these days. Intelligent social insurance is a basic interest and the quality of administration necessity of medicinal services application is low idleness or start to finish postponement of the wellbeing information from the purpose of cause to preparing an input. A few human services systems dependent on cloud and IoT have been accounted for since sensors create information and cloud can bolster assets required to store, process, and recover data on interest premises.

It is likewise recognized that IoT coordinated with the cloud is getting profound established with a wide selection of associated gadgets in the human services industry. Consequently, it is 
Journal of trends in Computer Science and Smart technology (TCSST) (2021)

Vol.03/ No. 01

Pages: $1-13$

https://www.irojournals.com/tcsst/

DOI: https://doi.org/10.36548/jtcsst.2021.1.001

especially evident that the series will be two-sided as the IoT including therapeutic gadgets and applications, and the other way around. This healthcare service, which significantly relies upon patient investment and subsequent information investigation by microchips and microcontrollers, is set to modify the manner in which wellbeing administrations are conveyed. In the medical services sector, IoT and cloud will continue to grow exponentially, advancing to a substantial and optimistic turnaround for all stakeholders.

\section{REFERENCES}

[1] Q. Zhang, L. Cheng, and R. Boutaba, "Cloud computing: state-of-the-art and research challenges," Journal of internet services and applications, vol. 1, no. 1, pp. 7-18, 2010.

[2] A. V. Dastjerdi, H. Gupta, R. N. Calheiros, S. K. Ghosh, and R. Buyya, "Fog computing: Principles, architectures, and applications," arXiv preprint arXiv:1601.02752, 2016.

[3] M. Hassanalieragh et al., "Health monitoring and management using Internet-of-Things (IoT) sensing with cloud-based processing: Opportunities and challenges," in Proc. IEEE Int. Conf. Services Comput.(SCC), Jun./Jul. 2015, pp. 285-292.

[4] A. Sawand, S. Djahel, Z. Zhang, and F. Naït-Abdesselam, "Multidisciplinary approaches to achieving efficient and trustworthy eHealth monitoring systems," in Proc. IEEE/CIC Int. Conf. Commun. China (ICCC), Oct. 2014, pp. 187-192.

[5] A. Koubaa and E. Shakshuki, "Cloud of Things: Integration of IoT with cloud computing," in Robots and Sensor Clouds, vol. 36. Cham, Switzerland: Springer, 2015, pp. 77-94.

[6] S. Distefano, G. Merlino, and A. Puliafito, "Enabling the Cloud of Things," in Proc. 6th Int. Conf. Innov. Mobile Internet Services Ubiquitous Comput. (IMIS), Jul. 2012, pp. 858-863.

[7] A. Botta, W. Donato, V. Persico, and A. Pescapé, "Integration of cloud computing and Internet of Things: A survey,' Future Gener. Comput. Syst., vol. 56, pp. 684-700, Mar. 2016.

[8] V. M. Rohokale, N. R. Prasad, and R. Prasad, "A cooperative Internet of Things (IoT) for rural healthcare monitoring and control," in Proc. 2nd Int.Conf.WirelessCommun.,Veh.Technol.,Inf.TheoryAerosp.Electron. Syst. Technol. (Wireless VITAE), Feb./Mar. 2011, pp. 1-6.

[9] G. Suciu, V. Suciu, and O. Fratu, "Big data processing for E-health applications using a decentralized cloud M2M system," Latest Trends Syst., vol. 2, pp. 232-237, 2013. 
Journal of trends in Computer Science and Smart technology (TCSST) (2021)

Vol.03/ No. 01

Pages: $1-13$

https://www.irojournals.com/tcsst/

DOI: https://doi.org/10.36548/jtcsst.2021.1.001

[10] M. Aazam, I. Khan, A. A. Alsaffar, and E.-N. Huh, "Cloud of Things: Integrating Internet of Things and cloud computing and the issues involved,' 'inProc.11thInt.BhurbanConf.Appl.Sci.Technol.(IBCAST), Jan. 2014, pp. 414419.

[11] E. Cavalcante et al., "On the interplay of Internet of Things and cloud computing: A systematic mapping study," Comput. Commun., vols. 89-90, pp. 17-33, Sep. 2016.

[12] S. M. Babu, A. J. Lakshmi, and B. T. Rao, "A study on cloud based Internet of Things: CloudIoT," in Proc. Global Conf. Commun. Technol. (GCCT), Apr. 2015, pp. 60-65.

[13] G. Suciu, V. Suciu, and O. Fratu, "Big data processing for E-health applications using a decentralized cloud M2M system,'” Latest Trends Syst., vol. 2, pp. 232-237, 2013.

[14] V. M. Rohokale, N. R. Prasad, and R. Prasad, "A cooperative Internet of Things (IoT) for rural healthcare monitoring and control," in Proc. 2nd Int.Conf.WirelessCommun.,Veh.Technol.,Inf.TheoryAerosp.Electron. $\quad$ Syst. $\quad$ Technol. (Wireless VITAE), Feb./Mar. 2011, pp. 1-6.

[15] V. M. Rohokale, N. R. Prasad, and R. Prasad, "A cooperative Internet of Things (IoT) for rural healthcare monitoring and control," in Proc. 2nd Int.Conf.WirelessCommun.,Veh.Technol.,Inf.TheoryAerosp.Electron. Syst. Technol. (Wireless VITAE), Feb./Mar. 2011, pp. 1-6.

[16] G. Fortino, A. Guerrieri, W. Russo, and C. Savaglio, "Integration of agent-based and Cloud Computing for the smart objects-oriented IoT," in Proc. IEEE 18th Int. Conf. Comput. Supported Cooper. Work Design (CSCWD), May 2014, pp. 493-498.

[17] Liane Margarida RockenbachTarouco, Leandro MárcioBertholdo et.al., "Internet of Things in healthcare: Interoperatibility and security issues", International Workshop on Mobile Consumer Health Care Networks, Systems and Services,2015.

[18] Felipe Fernandez, George C. Pallis," Opportunities and challenges of the Internet of Things for healthcare", EAI 4th International Conference on Wireless Mobile Communication and Healthcare (Mobihealth), 2014.

[19] Danilo F. S. Santos, Angelo Perkusich and Hyggo O. Almeida," Standard-based and Distributed Health Information Sharing form Health IoT Systems", IEEE 16th International Conference on e-Health Networking, Applications and Services (Healthcom), 2014. 
Journal of trends in Computer Science and Smart technology (TCSST) (2021)

Vol.03/ No. 01

Pages: $1-13$

https://www.irojournals.com/tcsst/

DOI: $\underline{\text { https://doi.org/10.36548/jtcsst.2021.1.001 }}$

[20] Alessio Botta, Walter de Donato, Valerio Persico, Antonio Pescap'e "On the Integration of Cloud Computing and Internet of Things", International Conference on Future Internet of Things and Cloud,2014.

[21] S. Luo and B. Ren, "The monitoring and managing application of cloud computing based on Internet of Things," Comput. Methods Programs Biomed., vol. 130, pp. 154-161, Jul. 2016.

[22] L. Catarinucci et al., “An IoT-aware architecture for smart healthcare systems, "IEEE InternetThingsJ.,vol.2, no.6,pp.515-526,Dec.2015.

[23] M. Aazam and E.-N. Huh, "Fog computing and smart gateway based communication for Cloud of Things," in Proc. Int. Conf. Future Internet Things Cloud (FiCloud), Aug. 2014, pp. 464-470.

[24] Y. Shi, G. Ding, H. Wang, H. E. Roman, and S. Lu, "the fog computing service for healthcare," in Proc. 2nd Int. Symp. Future Inf. Commun.Technol.UbiquitousHealthCare(Ubi-HealthTech),May2015, pp. 70-74.

[25] S. Sarkar, S. Chatterjee, and S. Misra, "Assessment of the suitability of fog computing in the context of Internet of Things," IEEE Trans. Cloud Comput., vol. 6, no. 1, pp. 46-59, Jan./Mar. 2018.

[26] F.Ramalho,A.Neto,K.Santos,J.B.Filho,andN.Agoulmine, "Enhancing eHealth smart applications: A Fog-enabled approach," in Proc. 17thInt.Conf.EHealthNetwo.,Appl.Services(HealthCom),Oct.2016, pp. 323-328.

[27] F. A. Kraemer, A. E. Braten, N. Tamkittikhun, and D. Palma, "Fog computinginhealthcareAreviewanddiscussion,'’IEEEAccess,vol.5, pp. 9206-9222, 2017.

[28] G. Aloi et al., "Enabling IoT interoperability through opportunistic smartphone-based mobile gateways,' J. Netw. Comput. Appl., vol. 81, pp. 74-84, Mar. 2017..

[29] Poulymenopoulou, Malamateniou, G. Vassilacopoulos," A virtual PHR authorization system", IEEE-EMBS International Conference on Biomedical and Health Informatics (BHI), 2014.

[30] Mankodiya, K., et al. "Wearable ECG module for long-term recordings using a smartphone processor." Proceedings of the 5th International Workshop on Ubiquitous Health and Wellness, Denmark. 2010. 